\title{
Determinação da Homogeneidade e Tendência das Precipitações na Bacia Hidrográfica do Rio Tapajós
}

\author{
Maria de Nazaré Alves da Silva ${ }^{1}$, Francisco Carlos Lira Pessoa ${ }^{2}$, \\ Rafaela Nazareth Pinheiro de Oliveira Silveira ${ }^{3}$, Gabrielle Souto Rocha ${ }^{3}$, \\ Daniel Alvino Mesquita ${ }^{3}$ \\ ${ }^{1}$ Departamento de Engenharia Civil, Faculdade de Tecnologia, Universidade Federal do \\ Amazonas, Manaus, AM, Brasil. \\ ${ }^{2}$ Programa de Pós-Graduação em Engenharia Civil, Faculdade de Engenharia Sanitária e \\ Ambiental, Universidade Federal do Pará, Belém, PA, Brasil. \\ ${ }^{3}$ PPrograma de Pós-Graduação em Engenharia Civil, Universidade Federal do Pará, Belém, PA, \\ Brasil.
}

\begin{abstract}
Resumo
O presente estudo determinou regiões homogêneas de precipitação média anual na bacia hidrográfica do Rio Tapajós por intermédio do método de agrupamento hierárquico de Ward, utilizando variáveis físico-climáticas como: a localização (latitude e longitude), a altitude e a precipitação média anual (PMA). Definiram-se 3 regiões homogêneas de precipitação, as quais foram validadas pelo índice de Silhouette. Aplicaram-se testes não-paramétricos (Mann Kendall, Spermann e o Estimador Sem) para analisar as tendências de precipitação em cada uma das regiões formadas. Os testes indicaram que existe tendência positiva nos dados de PMA na região composta pelas nascentes da bacia; por outro lado, na região central e próximo do exutório não ocorreram tendências positiva ou negativa nos dados monitorados, quer dizer, as séries possuem estacionariedade. A determinação dessas regiões homogêneas de precipitação é uma importante contribuição para o entendimento do comportamento climático na bacia hidrográfica, podendo auxiliar na gestão dos recursos hídricos.
\end{abstract}

Palavras-chave: análise de agrupamento, testes não-paramétricos, variabilidade climática.

\section{Determination of the Homogeneity and Tendency of Precipitations in the Tapajós River Basin}

\begin{abstract}
The present study determined homogeneous regions of mean annual precipitation in the Tapajós River basin, using Ward's hierarchical clustering method, using physical-climatic variables such as: location (latitude and longitude), altitude and mean annual precipitation (PMA). Three homogeneous regions of precipitation were defined, which were validated by the Silhouette index. Nonparametric tests (Mann Kendall, Spermann and Sen Estimator) were applied to analyze the precipitation trends in each of the formed regions. The tests indicated that there is a positive trend in the PMA data in the region composed by the sources of the basin, on the other hand, in the central and the exudation regions there were no positive or negative trends in the monitored data, that is, the series have statiomarity. The determination of these homogeneous regions of precipitation is an important contribution to the understanding of the climatic behavior in the river basin, and can help in the management of water resources.
\end{abstract}

Keywords: cluster analysis, nonparametric, climatic variability. 


\section{Introdução}

A precipitação é um importante componente de entrada do ciclo hidrológico, fundamental para a dinâmica dos meios físico e ambiental. Conhecer seu comportamento é indispensável, uma vez que qualquer alteração na sua quantidade e na distribuição espacial e temporal tem impacto significativo sobre as atividades humanas (agricultura, abastecimento de água, geração de energia elétrica e serviços urbanos diversos) e sobre os riscos naturais, tais como secas inundações e alagamentos (Dahria et al., 2016; Cruz et al., 2013).

A falta de monitoramento pluviométrico é um dos principais problemas que se apresentam em estudos de precipitação, pois impossibilita conhecer sua variabilidade ao longo do tempo e espacialmente nas bacias hidrográficas. Contudo, para atenuar a carência de informações, tem-se adotado diversas metodologias para a compreensão da precipitação em diferentes regiões.

Dentre inúmeras pesquisas que abordam a variabilidade climática, destaca-se a identificação de regiões hidrologicamente homogêneas de precipitação por intermédio da análise de agrupamentos - cluster analysis (Shirin e Thomas, 2015; Gonçalves et al., 2016; Farsadnia et.al., 2013; Hassan e Ping, 2012; Santos et al., 2015a) que, quando realizada de forma integrada, pode suprir a ausência de informações de um determinado local.

Em Hidrologia o termo regiões homogêneas, segundo Franchini e Suppo (1996), é definido como sendo aquelas regiões com similaridade hidrológica. A similaridade está relacionada com fatores físicos, climáticos e efeitos antrópicos da bacia que influenciam no comportamento da precipitação.

O método hierárquico de Ward é uma das técnicas de análise de agrupamentos que vêm se destacando na área de hidrologia, apresentando bons resultados na formação de grupos regionais homogêneos, como, por exemplo, nos estudos desenvolvidos por: Shirin e (2015), que classificaram, por meio do método de agrupamento hierárquico de Ward, o estado de Kerala, na İ́ndia, em zonas homogêneas com base na precipitação mensal de 63 estações pluviométricas por cinco anos consecutivos (2008-2012).

Já nos estudos de Hassan e Ping (2012) foram quantificados os padrões regionais de precipitação da bacia do LuanHe, com dados de precipitação anual de 17 estações fluviométricas para o período de 1932-1970, aplicando o método de análise de agrupamento de Ward e, para a validação dos grupos, o método L-momentos.

Por sua vez, Yang et al. (2010) utilizaram o método de Ward para determinar regiões homogêneas na China, tendo sido escolhidas quatro variáveis (latitude, longitude, altitude e precipitação média anual) das 42 estações pluviométricas selecionadas para descrever o regime de precipitação da região.

Enquanto que Farsadnia et.al. (2013) adotaram o método Selft-Organization Feature Map (SOM) com o
Fuzzy c-means, K-means e Ward para identificar regiões homogêneas de precipitação na província de Mazandaran, no norte do Irã.

No Brasil, especificamente na região amazônica, estudos semelhantes foram desenvolvidos, como o de Gonçalves et. al. (2016), que identificou regiões homogêneas de chuvas no estado do Pará utilizando dados de precipitação de 413 estações, bem como a relação das oscilações climáticas de grande escala considerando os fenômenos El Niño e La Niña.

Menezes et al. (2015) mostraram a possibilidade do estado do Pará ser dividido em três regiões pluviometricamente homogêneas, distribuídas em faixas zonais do sul para o norte. Santos et al. (2014(b)) determinaram subregiões homogêneas de precipitação na Amazônia brasileira, relacionando-as aos principais sistemas atmosféricos que afetam a chuva na região, com a qualidade dos grupos formados pelo método de Ward tendo sido validada por intermédio do índice de Silhouette, definindo 6 regiões homogêneas pluviais na Amazônia.

Assim como regionalizar as precipitações por meio do agrupamento em regiões homogêneas, as mudanças no comportamento da precipitação podem ser avaliadas por meio de métodos não-paramétricos, como a identificação de tendência na série de precipitações.

A análise de tendência da chuva é realizada aplicando-se os testes de Mann-Kendall, Rho de Spearman e o Estimador Sen's Slope. A aplicação desses testes foi observada nos estudos desenvolvidos por: Onyutha et al. (2016), que analisaram as tendências das chuvas em 39 localidades no rio Nilo, na África do Sul. Duncan et al. (2013), por sua vez, aplicaram os métodos não-paramétricos em séries temporais diárias de precipitação de 57 anos (195-2007) para o Nepal e discutiram a contribuição da tendência de precipitação para os recursos hídricos.

Anghileri et al. (2014) investigaram a relação dos testes estatísticos de tendência em séries temporais de precipitação e seus recursos hídricos nos Alpes italianos e suíços no período de 1974-2010. Tais autores quantificaram as tendências em diferentes estações pluviométricas, combinando o MASH (Moving Average over Shifting Horizon) com os testes de Mann-Kendall, de Kendall sazonal, de regressão linear e o método de Sen.

Keggenhoff et al. (2014) estudaram as tendências nos índices climáticos extremos na Geórgia, utilizando séries mínimas e máximas de temperatura e precipitações para o período de 1971 a 2010.

Sendo assim, este trabalho tem como objetivo identificar regiões homogêneas de precipitação na bacia hidrográfica do Rio Tapajós, por meio do método de agrupamento hierárquico de Ward, adotando características regionais de cada posto pluviométrico. Posteriormente, analisar a tendência da precipitação para cada uma das regiões formadas, utilizando os testes não-paramétricos de Mann-Kendall, Rho de Spearman e o Estimador Sen's Slope. 
De modo que o mapa das regiões homogêneas de precipitação resultante desta pesquisa poderá fornecer subsídios ao planejamento dos recursos hídricos na bacia hidrográfica do Rio Tapajós, de forma a reduzir o tempo de análise de dados e aumentando a confiabilidade dos resultados. Além de possibilitar a adoção do método em outras bacias hidrográficas do estado.

\section{Materiais e Métodos}

\section{1 Área de estudo e dados utilizados}

A bacia hidrográfica do rio Tapajós (Fig. 1) localizase entre as coordenadas $02^{\circ} 14^{\prime} 38^{\prime \prime}$ e $14^{\circ} 55^{\prime} 44^{\prime \prime} /$ Sul e $53^{\circ}$ $49^{\prime} 34^{\prime \prime}$ e $60^{\circ} 7^{\prime} 48^{\prime \prime} /$ Oeste, configura uma das principais sub-bacias do rio Amazonas, tendo como principais afluentes os Rios Juruena e Teles Pires. Além disso, possui uma área de drenagem de aproximadamente $492.000 \mathrm{~km}^{2}$, compreendida entre os estados do Amazonas (3\%), Pará (38\%) e Mato Grosso (59\%). Sua extensão é de aproximadamente $146 \mathrm{~km}$, englobando 45 sedes municipais, sendo 8 localizadas no estado do Pará e 37 no Mato Grosso (Santos et al., 2015).

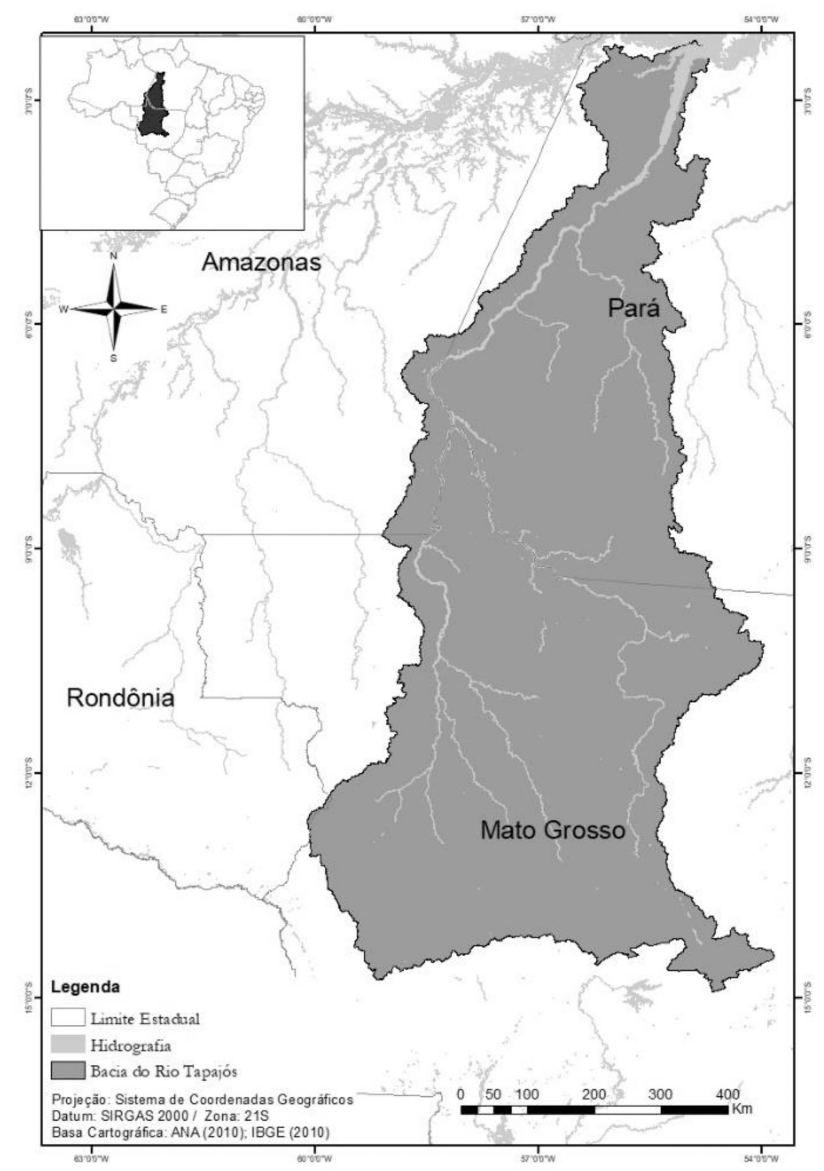

Figura 1 - Bacia hidrográfica do Rio Tapajós.
A bacia hidrográfica do Rio Tapajós é afluente da margem direita do rio Amazonas e contribui para a manutenção dos ecossistemas amazônicos, englobando áreas com potencial para a exploração de recursos naturais. Em estudo realizado por Sousa Júnior (2014) a estação de maior pluviosidade na bacia ocorre nos meses de dezembro a maio, sendo o mês de março o período mais chuvoso e o intervalo de junho a novembro como sendo o de menor quantidade de chuvas.

Para o desenvolvimento do estudo foram utilizados dados de 162 pontos de monitoramento da precipitação via satélite, espacializados em uma grade de $0,5 \times 0,5^{\circ}$ de latitude e longitude, obtidos no site do Centro de Pesquisa Climática da Universidade de Delaware-Newark, EUA, com a precipitação média anual $(\mathrm{mm})$ de cada ponto sendo interpolada por intermédio do algoritmo Shepard (Webber e Willmott, 1998).

Inicialmente foram obtidas imagens SRTM (Shuttle Radar Topography Mission) para determinar a área de drenagem da bacia e elaborar o Modelo de Elevação Digital (MDE) por meio do Software Geographic Information System (GIS). Em seguida, foram plotados os 162 pontos de monitoramento via satélite para determinar as variáveis climáticas (precipitação média anual) e topográfica (altitude, latitude e longitude).

Os dados de precipitação média anual - PMA (mm) correspondem ao período de 1960 a 1990 (31 anos), abrangendo, conforme recomendação da Organização Meteorológica Mundial - OMM (1994), uma série superior a 30 anos de informações para fins de confiabilidade na caracterização climática de uma região. É válido ressaltar que a utilização de dados via satélite foi priorizada em virtude da insuficiência de estações pluviométricas com séries de dados completas (acima de 30 anos) na bacia do Rio do Tapajós.

\subsection{Determinação das regiões homogêneas de precipitação (RHP)}

Para a determinação das regiões homogêneas de precipitação - RHP na bacia hidrográfica do Rio Tapajós foram utilizadas quatro variáveis: precipitação média anual $(\mathrm{mm})$, altitude $(\mathrm{m})$, latitude e longitude, que representam as características de cada um dos 162 pontos de monitoramento via satélite. Aplicou-se o método de agrupamento hierárquico de Ward com a finalidade de agrupar os indivíduos (pontos) com base em suas características, assim como uma medida de dissimilaridade denominada quadrado da distância euclidiana.

O método de Ward interliga as amostras por suas associações e considera, para a formação inicial dos grupos, aqueles indivíduos que proporcionam a menor soma de quadrados dos desvios. O método produz um dendograma, também chamado de árvore de decisão, no qual as amostras semelhantes são agrupadas entre si segundo as 
variáveis escolhidas, tendo sido aplicado em toda a série histórica de 31 anos.

O agrupamento foi feito por meio das somas dos quadrados dos desvios entre acessos ou a partir do Quadrado da Distância Euclidiana, verificando-se a relação pelas Eqs. (1)-(2):

$$
\begin{gathered}
S Q D_{i i^{\prime}}=\frac{1}{2} d_{i i^{\prime}}^{2} \\
S Q D_{i i^{\prime}}=\sum_{j=1}^{n} S Q D_{j\left(i i^{\prime}\right)}
\end{gathered}
$$

em que $S Q D_{j\left(i i{ }^{\prime}\right)}$ é a soma dos quadrados dos desvios para a $j$-ésima variável, considerando-se os postos $i$ e $i$; $d_{i i^{\prime}}^{2}$ o quadrado da distância euclidiana entre os postos $i$ e $i$, sendo $n$ o número de variáveis avaliadas. A soma dos quadrados dos desvios total é dada pela Eq. (3):

$$
S Q D T=\frac{1}{g} \sum_{i<}^{g} \sum_{i^{\prime}}^{g} d_{i i^{\prime}}^{2}
$$

\subsection{Validação dos agrupamentos}

$\mathrm{Na}$ validação do agrupamento final, empregou-se o índice de Silhouette, desenvolvido por Rousseeuw (1987). A largura da silhueta avalia a qualidade de uma solução do agrupamento, considerando tanto a compacidade (distância entre os pontos de dados dentro do mesmo grupo) quanto a separação (distância entre os pontos de dados em dois grupos vizinhos). Esse método possibilita determinar o número apropriado de grupos, tal que o valor de $k$ é escolhido de maneira que forneça o melhor valor médio do Silhouette (Gil et al., 2015; Santos et al., 2015b), Eqs. (4).

$$
s(i)=\frac{b_{i}-w_{i}}{\max \left(b_{i}, w_{i}\right)}
$$

Para $b_{i}=\min _{k}\left(B_{i, k}\right)$ em que $w_{i}$ é a distância média do $i$ ésimo ponto até os outros pontos de um mesmo grupo e $B_{(i, k)}$ é a distância média do $i$-ésimo ponto até os pontos de outro grupo $k$.

Quando o índice apresenta um valor unitário positivo, os pontos estão dispostos de maneira adequada. Por outro lado, ao ser caracterizado pelo valor zero, se torna impossibilitada a identificação do grupo ao qual pertencem. Por fim, para um valor unitário negativo, os pontos provavelmente estão nos grupos errados.

A largura média da silhueta de um grupo - $-s(k)$ para todo $i$ em um dado grupo é definida como a média de todas as silhuetas individuais, em que $n$ é o número de objetos no conjunto de dados, conforme Eq. (5):

$$
\bar{s}(k)=\frac{1}{n} \sum_{i=1}^{n} s(i)
$$

Após a identificação das RHP, realizou-se a análise de tendência da precipitação média anual para cada uma das regiões formadas. Utilizou-se os testes não-paramétricos de Mann Kendall (MK), Rho de Spearman e o Estimador Sen's Slope (Sen).

\subsection{Teste de Mann-Kendall}

O teste de Mann Kendall foi utilizado para identificar eventuais flutuações nas tendências ao longo do tempo e para detectar os possíveis pontos de mudança na série de chuvas, conforme estudo desenvolvido por Bari et al. (2016) na região norte de Blagandesh. Dessa forma, inicialmente foram desenvolvidas a estatística e sua variância, dado por "var (S)". A estatística padronizada Z é então calculada usando o desvio padrão $(\mathrm{S})$ e a variância $\operatorname{var}(\mathrm{S})$, conforme Eq. (6):

$$
Z= \begin{cases}\frac{S-1}{\sqrt{\operatorname{var}(S)}} & \text { se } S>0 \\ 0 & \text { Se } S=0 \\ \frac{S+1}{\sqrt{\operatorname{var}(S)}} & \text { se } S<0\end{cases}
$$

Sendo a variância calculada pela Eq. (7):

$$
\operatorname{Var}(S)=\frac{n(n-2)(n 2+5)-\sum_{i=1}^{p} t i(i)(i-1)(2 i+5)}{18}
$$

em que: $n$ é o comprimento da série de tempo; $p$ número de valores amarrados em $X$ e $t$ o número de laços de extensão $i$.

Por meio da análise da estatística z, se aceita a hipótese nula $\left(H_{\mathrm{o}}\right)$ quando os dados se apresentarem estáveis. Em contrapartida, haverá rejeição a favor da hipótese alternativa $\left(H_{\mathrm{a}}\right)$ quando configurada a tendência nos dados.

Valores positivos de $Z$ indicam tendências crescentes e valores negativos mostram tendências decrescentes. Ao testar as tendências de aumento ou diminuição no nível de significância $\alpha, H_{\mathrm{a}}$ será rejeitada por um valor absoluto de $Z$ maior que $Z_{1}-\alpha / 2$ obtido a partir das tabelas de distribuição normal.

\subsection{Teste de Rho de Spearman}

Este teste baseia-se na classificação do coeficiente de correlação de Spearman $\left(\mathrm{r}_{\mathrm{s}}\right)$ definido por Darmen e Hall (1990) como visto na Eq. (8):

$$
r s=1-\frac{\left(6 * \sum_{i=1}^{n} d_{i}^{2}\right)}{[n *(n * n-1)]}
$$

No qual $n$ é o número total de dados, $D$ é diferença e $i$ é a ordem cronológica. A diferença entre os rankings é calculada pela Eq. (9): 


$$
D i=K x i-K y i
$$

em que Kxi é a posição da variável e $x i$ é o número de ordem cronológica de observações.

A série de observações yié transformada em seu equivalente, Kyi atribuindo-se ao número de ordem cronológica de uma observação na série original o número de ordem correspondente na série classificada yi. Se houver duas ou mais observações yi com o mesmo valor, a convenção é tomar Kxi como a classificação média. Pode-se testar a hipótese nula $H o: r_{s}=0$ (não há tendência) em face da hipótese alternativa $\mathrm{Ha}: r_{s}<>0$ (há uma tendência) por meio da estatística do teste, conforme a Eq. (10):

$$
t_{t}=r s \sqrt{\operatorname{Var}[r s]}
$$

A $[r s]$ é determinada pela Eq. (11).

$$
\operatorname{Var}[r s]=1 n-2
$$

na qual $t$ tem a distribuição $t$ de Student $\operatorname{com} v=n-2$ graus de liberdade.

A distribuição Student - $t$ é simétrica em torno de $t=0$. Caso exista relação, a convenção é considerar a classificação média. A hipótese nula é testada pelo cálculo do teste estatístico, $t_{c a l}$ Eq. (12):

$$
t_{c a l}=r s \sqrt{\frac{n-2}{1-r s^{2}}}
$$

em que $t_{c a l}$ é a Distribuição Student-t, com $V=n-2$ graus de liberdade. Em um nível de significância a série de tempo não tem tendência se $-Z \alpha / 2<$ tcal $<Z \alpha / 2$. Para o critério de validação da correlação ser estatisticamente significativo, o $\left|t_{c a l}\right|$ deve ser $\geq t_{t}$.

\subsection{Estimador Sen's slope (Sen)}

As estatísticas $Z$ são utilizadas para testar a presença de uma tendência estatisticamente significativa em comparação com a probabilidade tolerável. Neste estudo o nível de significância padrão é definido como 0,05 . Se uma tendência linear está presente em uma série temporal, então a inclinação verdadeira (variação por unidade de tempo) pode ser estimada utilizando-se um teste não-paramétrico desenvolvido por Sen (1968). A estimativa da inclinação de $\mathrm{N}$ pares de dados é computada pela Eq. (13):

$$
Q i=X j-X k j-k
$$

para $\mathrm{i}=1,2, \ldots, \mathrm{N}$ em que $X j$ e $X k$ são valores dos dados no tempo j e $\mathrm{k}(\mathrm{j}>\mathrm{k})$ respectivamente, sendo que a mediana dos N valores de $Q i$ é o estimador da inclinação de Sen. Quando N é ímpar, o estimador de Sen é calculado pela Eq. (14); se for par, será calculado pela Eq. (15):

$$
\begin{gathered}
\text { Qmed. }=Q[N+1 / 2] \\
\text { Qmed. }=12(Q[N / 2]+Q N+2 / 2)
\end{gathered}
$$

O teste de Qmed. é realizado por intermédio de um teste bilateral a nível de $100(1-\alpha) \%$ de intervalo de confiança dado pela Eq. (17):

$$
I C a=Z 1-\alpha / 2 \sqrt{\operatorname{Var}_{[r s]}}
$$

Sendo $\operatorname{Var}_{[r s]^{-}}$definida na Eq. (11), com $Z_{1}-\alpha / 2$ sendo determinado por meio da tabela da distribuição normal padrão, bem como calculados os limites inferior e superior do intervalo de confiança.

\section{Resultados e Discussão}

O resultado da análise estatística descritiva para as variáveis PMA e altitude é apresentado na Tabela 1, onde se observa que a menor altura de PMA para todos os 162 pontos de monitoramento foi de $1.613 \mathrm{~mm}$, sendo os valores de 2.480,8 e 2.092,6 mm referentes, respectivamente, à maior altura e a sua média. Os menores valores de PMA foram encontrados nos pontos mais próximos das cabeceiras, no estado do Mato Grosso, os quais correspondem às maiores altitudes. Em contrapartida, os valores de altitude mínimo, máximo e médio são, na devida ordem, 21,5, 598,8 e 281,6 m.

A amplitude interquartílica dos valores PMA, que engloba $50 \%$ dos dados centrais, está compreendida entre $1.875,0$ e $2.304,0 \mathrm{~mm}$. Ademais, o intervalo de 1.613,0 a $1.875,0 \mathrm{~mm}$ equivale a $25 \%$ das PMA mais baixas, ao passo que as mais altas variaram de 2.304,0 a 2.480,8 mm, conforme pode ser visto na Fig. 2 (a) e (b).

Nota-se na Fig. 2 (a) e (b) a inexistência de valores discrepantes (outliers) de PMA e de altitudes.

Na determinação das RHP para a bacia do Tapajós foi utilizado o método hierárquico de Ward, considerandose como observações os 162 pontos de monitoramento via satélite. O quadrado da distância euclidiana foi usado

Tabela 1 - Estatística descritiva para os 162 pontos de monitoramento.

\begin{tabular}{lcc}
\hline Estatística descritiva & PMA $(\mathrm{mm})$ & Altitude \\
\hline $\mathrm{N}^{\circ}$ de observações & 162 & 162 \\
Mínimo & $1.613,0$ & 21,5 \\
Máximo & $2.480,8$ & 598,8 \\
$1^{\circ}$ Quartil & $1.875,0$ & 190,7 \\
Mediana & $2.139,0$ & 271,6 \\
$3^{\circ}$ Quartil & $2.304,0$ & 373,2 \\
Média & $2.092,6$ & 281,6 \\
Variância (n-1) & $65.328,0$ & $18.656,7$ \\
Desvio-padrão (n-1) & 255,6 & 136,6 \\
\hline
\end{tabular}


como medida de similaridade. As variáveis utilizadas como dados de entrada são: PMA, altitude, latitude e longitude.

Os grupos foram determinados por intermédio de um corte transversal no dendograma, como pode ser visualizado na Fig. 3, com base no critério matemático de inércia (distância entre os grupos), de forma que quanto menor o valor do quadrado da distância euclidiana, maior será a similaridade entre o conjunto de pontos dentro de um grupo e menor será a semelhança entre os grupos formados. A linha horizontal do dendograma representa os pontos e a linha vertical, o quadrado da distância euclidiana.

Analisando o dendograma, o qual foi cortado a uma distância igual a 4, observa-se a formação de 3 grupos distintos da direita para a esquerda, lembrando que essa decisão é subjetiva. No caso, cada grupo representa uma região hidrologicamente homogênea de precipitação.

A Tabela 2 apresenta as características de cada uma das 3 regiões formadas por meio do método de Ward.

$\mathrm{Na}$ Tabela 2 percebe-se que a variável PMA apresentou limites bem definidos entre as 3 regiões formadas, tendo sido os maiores valores de altura de precipitação encontrados na RH1, composta por 78 pontos analisados, o que representa mais de $48 \%$ dos dados utilizados. Já as menores precipitações estão na RH3, com 31 dos pontos estudados, pouco menos de $20 \%$ do total. A RH2, formada por 53 pontos $(32,72 \%)$, pode ser considerada uma área intermediária com valores de precipitação próximos da média geral igual a 2.092,6 mm.

A variável altitude apresenta-se de forma desordenada entre as regiões, razão pela qual não foi possível identificar intervalos superiores e inferiores representativos para cada região. Entretanto, se forem levados em consideração os valores médios das altitudes em cada grupo, o cenário melhora, uma vez que as médias das
Tabela 2 - Características de cada região homogênea de precipitação.

\begin{tabular}{lcccc}
\hline RHP & $\mathrm{N}^{\circ}$ de pontos & $\%$ & PMA (mm) & Altitude (m) \\
\hline RH 1 & 78 & 48,15 & $2.173,2-2.480,8$ & $21,5-345,2$ \\
RH 2 & 53 & 32,72 & $1.820,5-2.155,1$ & $40,5-587,4$ \\
RH 3 & 31 & 19,13 & $1.613,0-1.799,5$ & $309,8-598,8$ \\
\hline
\end{tabular}

RH1, RH2 e RH3 são, respectivamente, 206,1, 286,6 e $449,2 \mathrm{~m}$.

Após a utilização do método de Ward, o índice Silhouette foi aplicado para validar os grupos (RHP) formados. Este índice sinaliza boa qualidade na formação dos grupos quando o seu valor é maximizado, quer dizer, quanto mais próximo de 1 , melhor.

A Fig. 4 apresenta os valores do índice de $\mathrm{Si}$ lhoeuttes(i) encontrados para cada ponto de monitoramento estudado, assim como os valores médios para cada um dos 3 grupos definidos.

Analisando a Fig. 4, nota-se que os resultados obtidos com a aplicação do Índice de Silhouette são satisfatórios, uma vez que a largura média da Silhouette é igual a 0,75 . Santos et al. (2015) encontraram valores de Índice de Silhouette inferiores a 0,50 para identificar sub-regiões de precipitação na Amazônia.

Observa-se também que os valores médios $-s(k)$ de cada grupo - grupo $1(0,75)$, grupo $2(0,60)$ e grupo 3 $(0,90)$ - apresentaram bons resultados, indicando que todos os elementos (pontos) estão localizados em seus respectivos grupos.

Sendo assim, as 3 RHP obtidas por meio do método de Ward e o relevo da bacia hidrográfica do rio Tapajós estão demostrados espacialmente nos mapas da Fig. 5 (a) e (b).

A RH 3 é formada por pontos de monitoramento que apresentam precipitações com média de 1.700,2 mm,

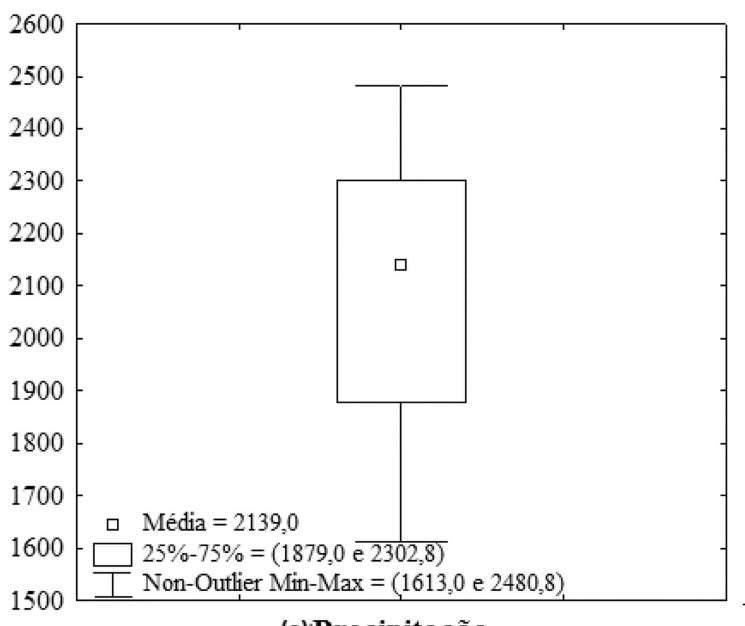

(a):Precipitação

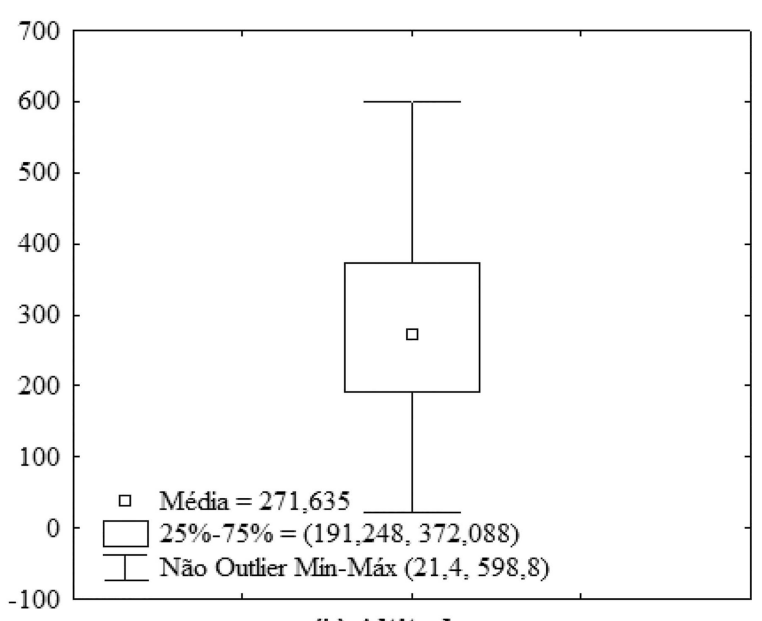

(b) Altitude

Figura 2 - Box plot das variáveis PMA (a) e altitude (b) para o período de 31 anos. 


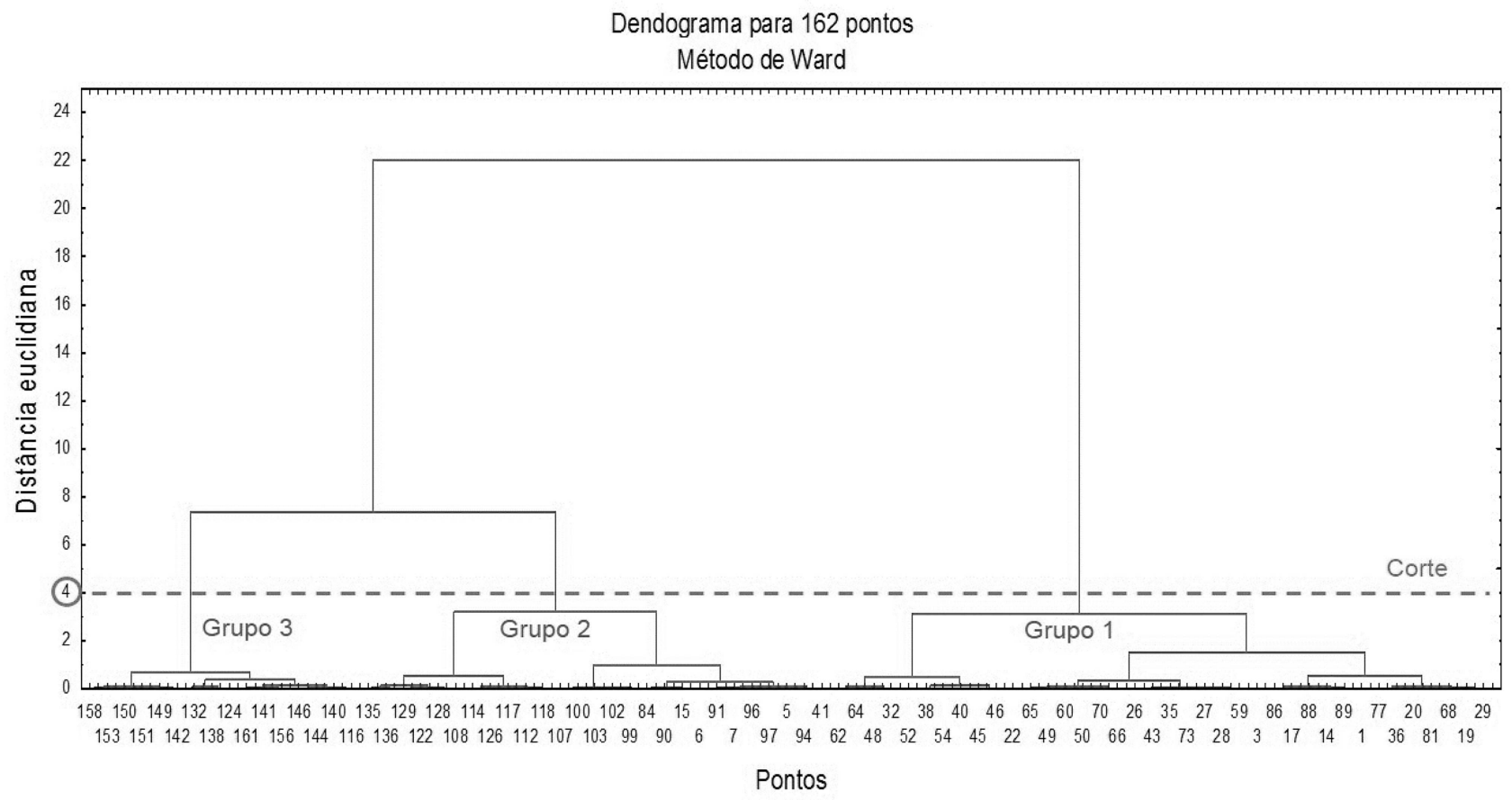

Figura 3 - Dendograma de análise de agrupamento para os 162 pontos de monitoramento da precipitação.

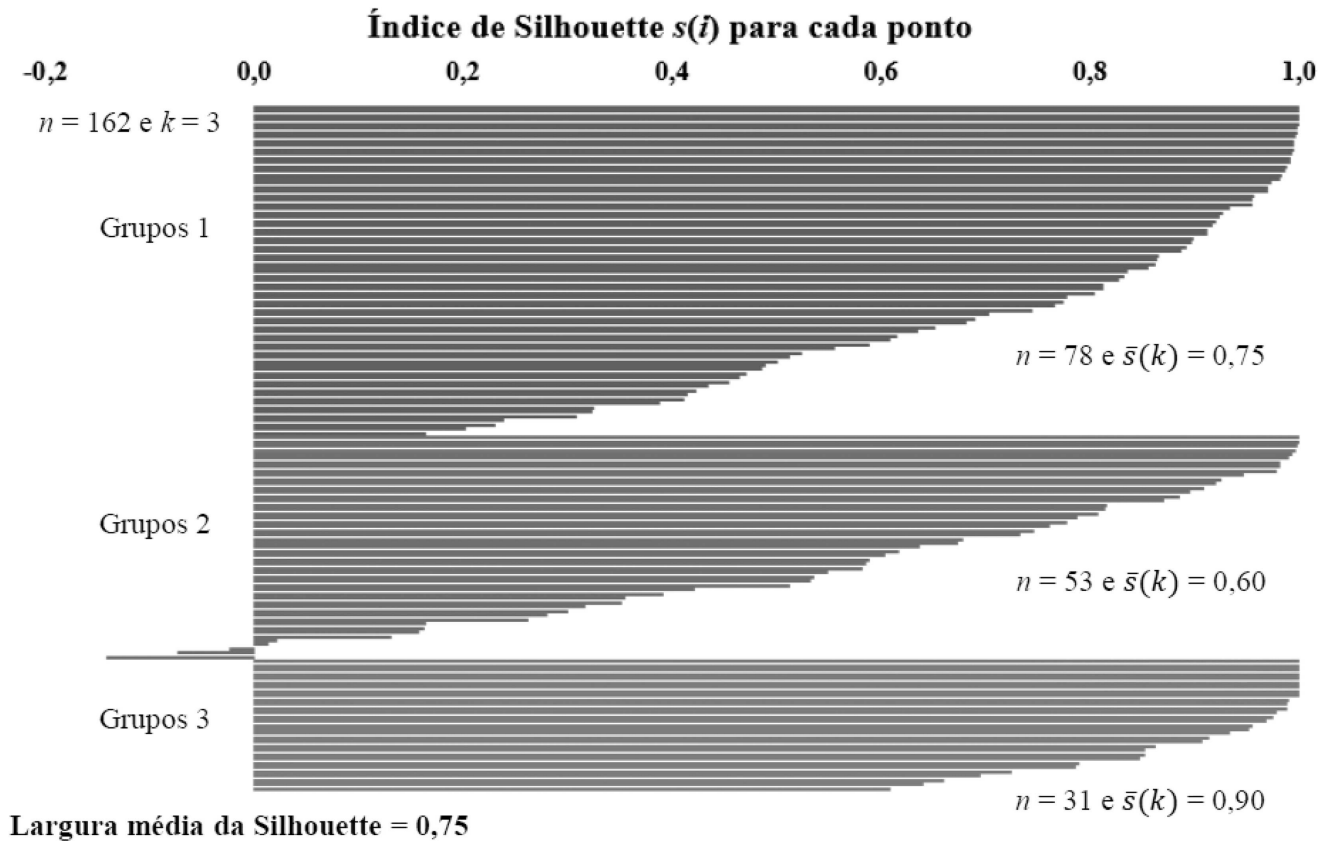

Figura 4 - Valores do Índice de Silhouette para os 3 grupos (RHP) formados.

mínima de 1.613,0 mm e máxima de 1.799,5 $\mathrm{mm}$. Esses pontos estão concentrados nas sub-bacias do alto Tapajós, onde predomina o bioma do cerrado de clima tropical com baixo índice pluviométrico. De acordo com Domingues e Bermann (2012), o sul da bacia do rio Tapajós é coincidente com a área definida como Arco do Desmatamento, fator que favorece a diminuição da altura de chuvas na região. De uma área de drenagem de $492.000 \mathrm{~km}^{2}$, a RH 3 corresponde a $19 \%\left(94.000 \mathrm{~km}^{2}\right)$ da bacia, com altitudes variando entre 309,8 a $598,8 \mathrm{~m}$, onde o relevo é o mais elevado.

Já na RH1 que equivale a 33,3\% $\left(163.000 \mathrm{~km}^{2}\right)$ da área de drenagem da bacia, ocorrem as maiores precipitações, com uma média de $2.318,2 \mathrm{~mm}$, máxima de 


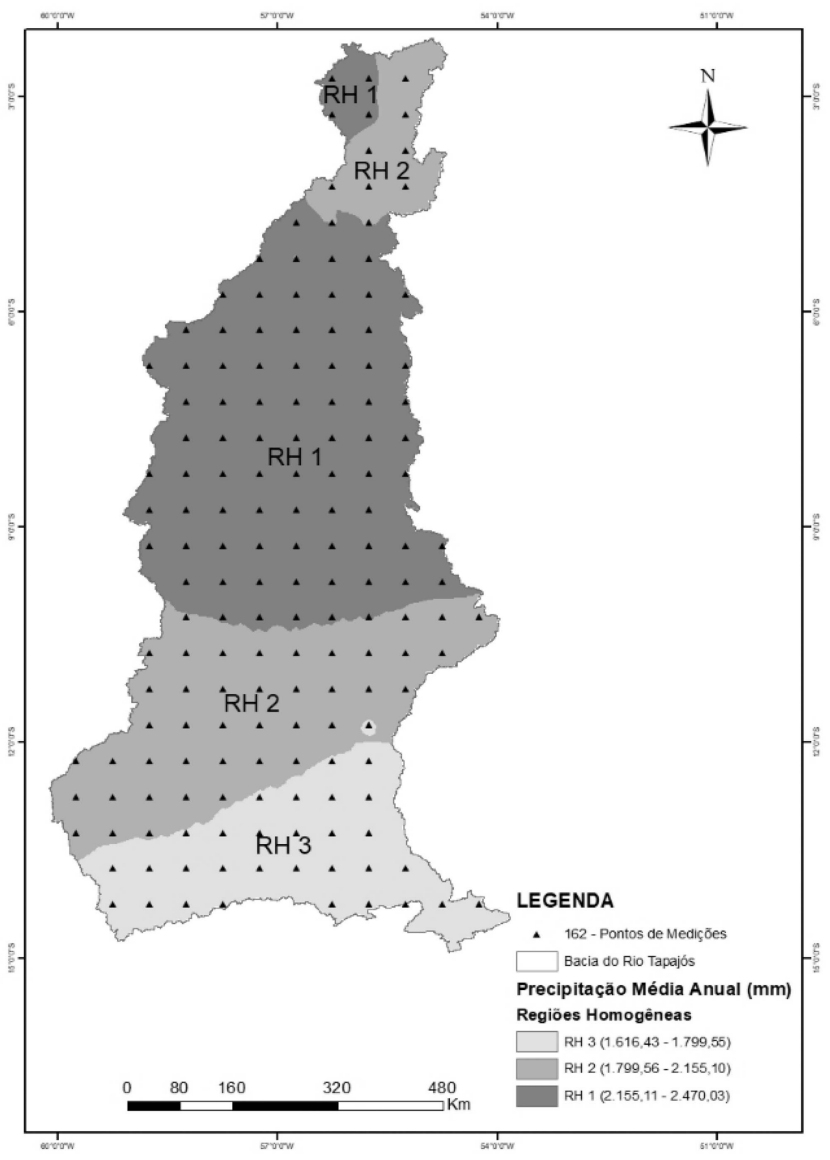

(a)

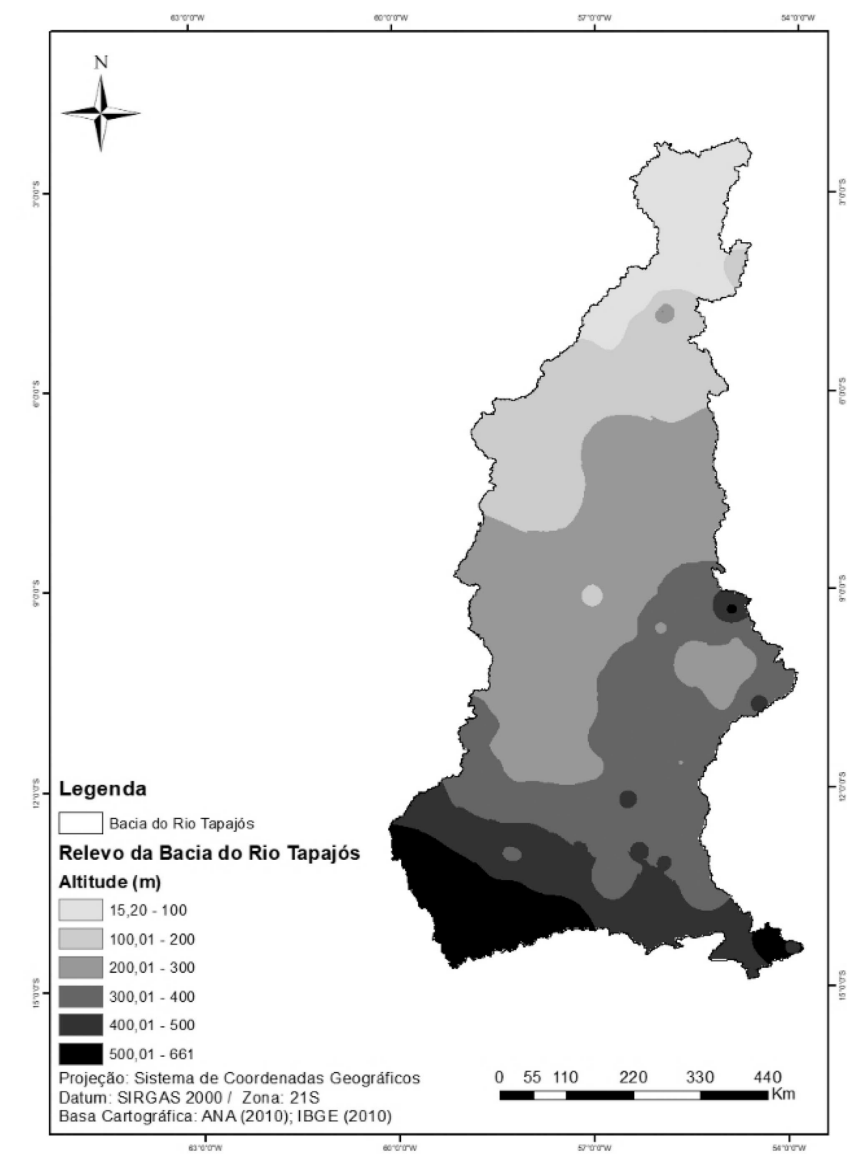

(b)

Figura 5 - Mapa das regiões homogêneas de precipitação (a) e mapa do relevo da Bacia Hidrográfica do Tapajós (b).

$2.480,8 \mathrm{~mm}$ e mínima de $2.173,2 \mathrm{~mm}$. A região localiza-se na porção central em direção à foz da bacia (porção norte), apresentando a menor média de altitude $(206 \mathrm{~m})$. No extremo norte da bacia a região apresenta altitudes ainda menores (21,5 a $62,3 \mathrm{~m})$, o que significa dizer que o relevo é mais plano.

A RH2 localiza-se na porção central em direção ao sul da bacia e representa 47,7\% $\left(235.000 \mathrm{~km}^{2}\right)$ da área de drenagem, com altitude média de 286,6 m. É formada por pontos com média de precipitações anuais de aproximadamente $1.990,1 \mathrm{~mm}$, com mínima de $1.820,5 \mathrm{~mm}$ e máxima de $2.155,1 \mathrm{~mm}$.

Na RH1 há predominância do bioma amazônico, abrigando grande parte das áreas protegidas (unidades de conservação e de proteção). Por outro lado, na RH2 e em parte da RH3 concentram-se as ocupações urbanas.

De forma geral, a bacia hidrográfica do Rio Tapajós tem uma extensa malha hidroviária e concentra grandes e pequenas centrais hidrelétricas, assim como áreas de mineração e portos graneleiros. Essas atividades influenciam direta e indiretamente no comportamento da precipitação da região, principalmente no que diz respeito ao plantio de soja (com destaque o Arco do Desmatamento) e da agropecuária.

O conhecimento do comportamento das precipitações, por meio dessas regiões homogêneas na bacia do Tapajós, poderá servir como ferramenta de gestão e planejamento dos recursos hídricos nesta bacia, permitindo analisar a disponibilidade da água de precipitação para atender ou não às atividades existentes na região.

As RHP determinadas pelo método de Ward apresentaram diferentes padrões de precipitações entre os grupos e similaridade dentro de cada grupo, o que permitiu avaliar as anomalias ocorridas nos 31 anos das séries, assim como a influência das mesmas no acréscimo ou decréscimo das precipitações.

As Figs. 6 and 7 e 8 apresentam o comportamento da precipitação total anual - PTA para cada uma das três regiões homogêneas formadas. A PTA representa o somatório de todos os valores de PMA de cada ponto de monitoramento pertencente as suas respectivas regiões.

Na Fig. 6 pode ser observado que, para os anos de 1960, 1962, 1964, 1985 e 1989, as PTA apresentaram valores superiores à média contida no gráfico, assim como 
ao limite superior do desvio padrão para toda a série. Esses anos podem ser considerados como anormais, com valores elevados de altura de precipitação, principalmente nos anos de 1964 e 1985, uma vez que ocorreu o fenômeno La Niña com intensidade moderada.

Por sua vez, nos anos de 1963, 1976, 1981, 1983 e 1987 os valores PTA ficaram abaixo do limite inferior do desvio padrão da série, tendo sido considerados anos anormais em decorrência do fenômeno El Niño, com baixa intensidade pluviométrica.

A RH 2 apresenta comportamento mais estável para as PTA, como pode ser observado na Fig. 7, em que nos anos de 1963, 1966, 1967, 1970 e 1983 os valores ficaram abaixo do limite inferior do desvio padrão da série, em virtude do fenômeno El Niño. Por outro lado, nos anos de La Niña (1974 e 1989) a precipitação foi superior ao desvio padrão da série e a linha de tendência se manteve estável em relação à média da PTA.
Analisando a Figura 8, nota-se que na RH3 o comportamento das PTA sofreu interferência direta do fenômeno El Niño, em especial no ano de 1969, quando o valor da PTA foi o menor de toda a série de 31 anos. Em contrapartida, no ano de 1980 o valor da PTA foi superior ao limite do desvio padrão, porém não ocorreram anomalias nesse período, podendo ser caracterizado como um ano atípico. Percebe-se que há um crescimento na linha de tendência, mas não chega a ser considerado fora do padrão de intensidade das precipitações.

Essas anomalias que ocorreram ao logo dos 31 anos têm grande contribuição na caracterização da ocorrência de tendência da PMA, podendo ser evidenciadas por meio dos testes não-paramétricos de Mann Kendall, Spearman e o Estimador Sen para um nível de significância de 5\%. A Tabela 3 apresenta os resultados encontrados por intermédio dos métodos não-paramétricos aplicados para cada uma das RHP definidas pelo método de Ward.

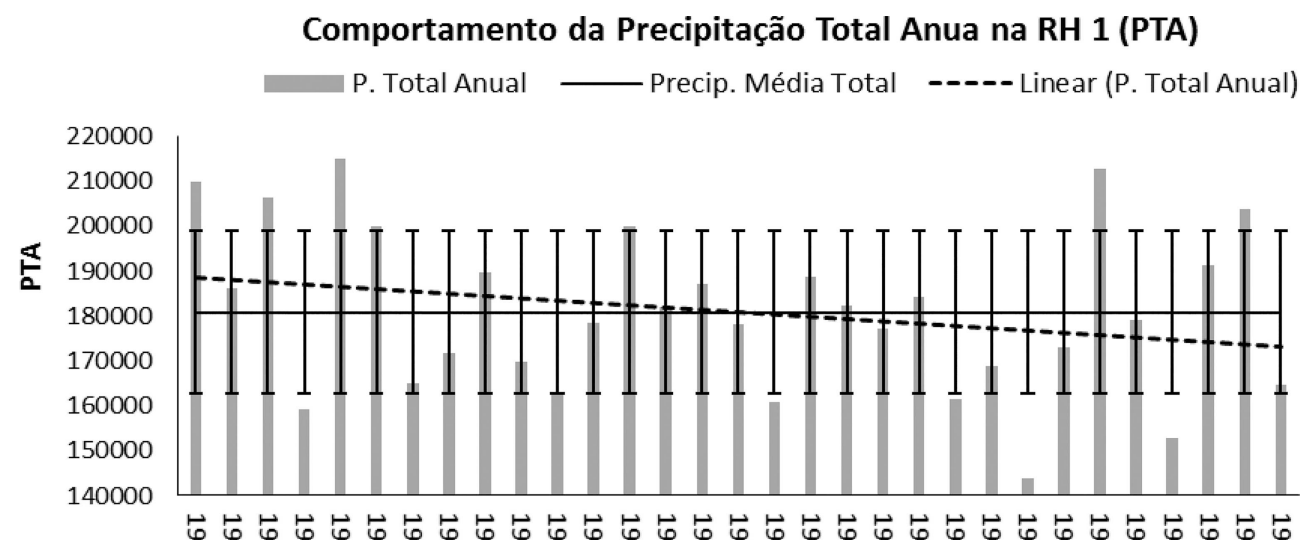

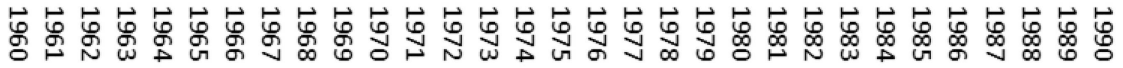

Anos

Figura 6 - Comportamento da PTA para a RH1.

\section{Comportamento da Precipitação Total Anual na RH 2 (PTA)}

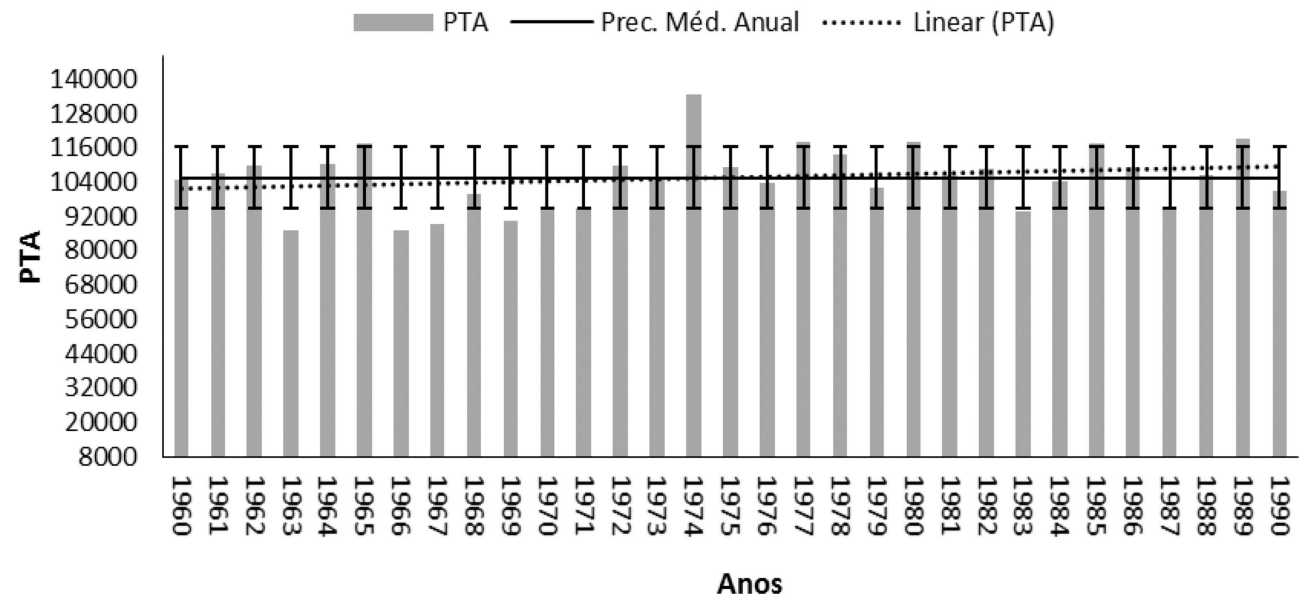

Figura 7 - Comportamento da PTA para a RH2. 


\section{Comportamento da Precipitação Total Anual na RH 3 (P'TA)}

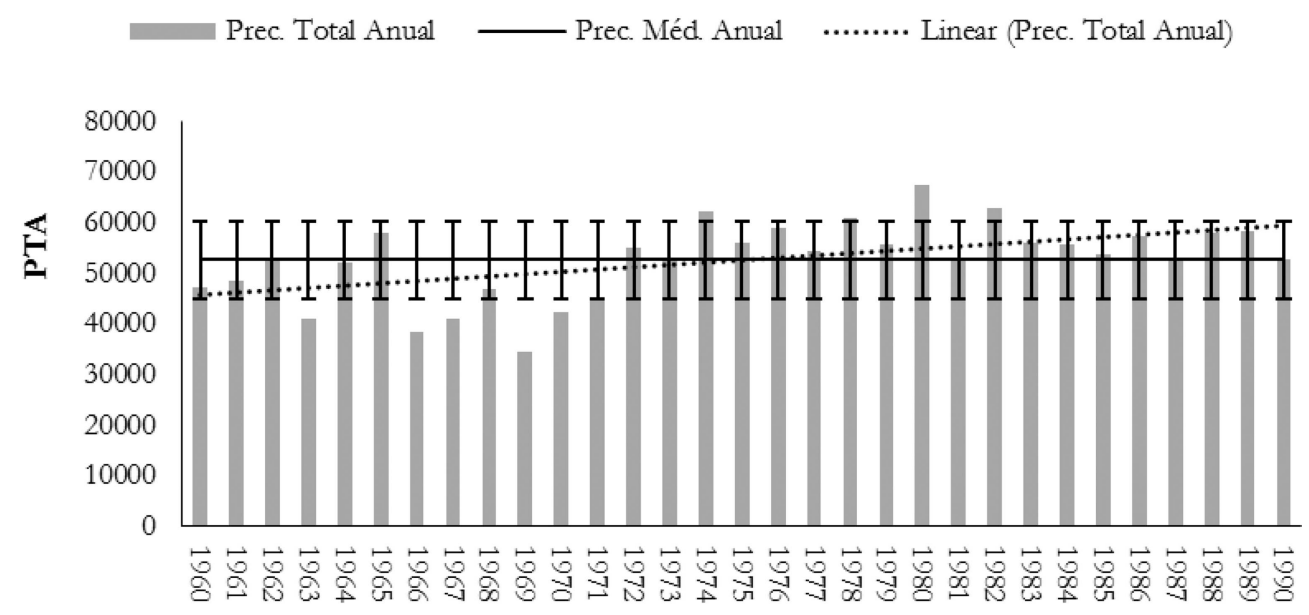

Anos

Figura 8 - Comportamento da PTA para a RH 3.

Tabela 3 - Resultados dos testes não paramétricos Mann Kendall, Spearman e o Estimador Sen.

\begin{tabular}{|c|c|c|c|c|c|c|}
\hline \multirow[t]{2}{*}{ RHP } & \multirow{2}{*}{$\begin{array}{c}\text { MK } \\
\text { Z }\end{array}$} & \multicolumn{2}{|c|}{ Spearmann } & \multirow[t]{2}{*}{$\operatorname{Sen}(\mathrm{mm})$} & \multirow[t]{2}{*}{ Hipótese } & \multirow[t]{2}{*}{ Tendência } \\
\hline & & $\mathrm{t}_{\mathrm{cal}}$ & $t_{t}$ & & & \\
\hline RH 1 & $-1,293$ & $-1,249$ & $-1,217$ & $-7,540$ & Ho & Não há tendência \\
\hline RH 2 & 1,173 & 1,042 & 1,023 & 5,917 & Ho: Nula & Não há tendência \\
\hline RH 3 & 2,893 & 2,560 & 2,930 & 12,509 & Ha: Positiva & Crescente \\
\hline
\end{tabular}

Nota-se que a PMA não apresentou tendências significativas nas RH1 e RH2, pois a hipótese nula foi confirmada por intermédio dos métodos não-paramétricos (Mann Kendall e Spearmann) para um nível de significância de 5\%. Todavia, observa-se uma tendência negativa de $-7,54 \mathrm{~mm} /$ ano na RH1 e uma tendência positiva de 5,92 mm/ano na RH2.

Os testes não-paramétricos aplicados à série de PMA revelaram um acréscimo de 12,51 mm/ano de altura de precipitação para a RH3, indicando uma tendência positiva. Por conseguinte, constata-se que o comportamento da PMA vem tendo um acréscimo considerável na sua altura nas nascentes da bacia hidrográfica do Rio Tapajós. Por outo lado, na parte central e no exutório da bacia ocorreu tanto acréscimo quanto decréscimo na altura da PMA, porém essas variações na precipitação não apresentam grande relevância.

Resultados similares com aumento da precipitação (tendência positiva) entre os meses de julho e dezembro no estado do Mato Grosso, Goiás e região do Nordeste foram encontrados na pesquisa desenvolvida por Salviano et al. (2016).

De acordo com Limberger e Silva (2016), o El Niño - Oscilação Sul é o principal fenômeno que influencia na variabilidade da precipitação na bacia amazônica, razão pela qual o decréscimo da chuva em algumas áreas da região pode ser consequência deste fenômeno. Em contrapartida, Silveira et al. (2017) avaliaram a tendência de precipitação no distrito de Abunã - Porto Velho - Rondônia, tendo concluído que os eventos climáticos (El Niño e La Niña) influenciaram no aumento da precipitação na região.

\section{Conclusão}

Pelos resultados obtidos nesse estudo, conclui-se que o método de agrupamento hierárquico de Ward e o índice de Silhouette são satisfatórios e adequados para a formação e a validação de regiões homogêneas de precipitação. A aplicação eficiente dessas metodologias confirma-se pela delimitação de 3 grupos (regiões homogêneas) distintos, com precipitações médias anuais bem definidas, tal qual a espacialização das regiões condizente com as alturas de chuvas registradas na bacia hidrográfica do Rio Tapajós.

Os testes não-paramétricos (Mann Kendall, Spearman e Estimador Sen) indicaram um aumento - tendência positiva - nas séries de precipitação pertencentes aos pontos de monitoramento próximos das nascentes da bacia do Tapajós. Esses resultados apontam que mudanças estão 
ocorrendo no comportamento da precipitação, podendo ser associadas aos fenômenos El Niño e La Niña.

A determinação dessas regiões homogêneas de precipitação, além de contribuir para a compreensão da variabilidade climática nessa região, poderá servir como ferramenta de apoio para a gestão e o planejamento dos recursos hídricos na bacia hidrográfica do Rio Tapajós.

\section{Referências}

ANGLILERI, D.; PIANOSI, F.; SESSA, R.S. Trend detection in seasonal data: from hydrology to water resources. Journal of Hydrology, v. 511, p. 171-179, 2014.

BARI, S.H.; MUHAMMAD, T.U.; MD, A.H.; HUSSAIN, M. Analysis of seasonal and annual rainfall trends in northern Bangladesh. Atmospheric Research, v. 176-177, p. 148$158,2016$.

DARMEN, E.R.; HALL, M.J. Screening of Hydrological Data: Tests for Stationarity and Relative Consistency. International Institute for Land Reclamation and Improvement, 1990. http://edepot.wur.nl/71119.

DUNCAN, J.M.A.; BIGGS, E.M.; DASH, J.; ATKINSON, P.M. Spatio-temporal trends in precipitation and their implications for water resources management in climate-sensitive Nepal. Applied Geography, v. 43, p. 138-146, 2013.

FARSADNIA, F.; KAMROOD, M.R.; MOGHADDAM NIA, A.; MODARRES, R.; BRAY, M.T.; HAN, D.; SADATINEJAD, J. Identification of homogeneous regions for regionalization of watersheds by two-level self-organizing feature maps. Journal of Hydrology, v. 509, p. 387-397, 2013.

FRANCHINI, M.; SUPPO, M. Regional analysis of flow duration curves for a limestone region. Water Resource Management, Dordrecht, Netherlands, v. 10, p.199-218, 1996.

GIL, V.O.; FERRAI, F.; EMMENDORFER, L. Investigação da aplicação de algoritmos de agrupamento para o problema astrofísico de classificação de galáxias. Revista Brasileira de Computação Aplicad, v. 7, n. 2, p. 52-61, 2015.

GONÇALVES, M.F.; BLANCO, C.J.C.; SANTOS, V.C.; OLIVEIRA, L.L.S.; PESSOA, F.C.L. Identification of Rainfall Homogenous Regions taking into account El Niño and La Niña and Rainfall Decrease in the state of Pará, Brazilian Amazon. Acta Scientiarum. Technology. v. 38, n. 2, p. 209-216, 2016. http://dx.doi.org/10.4025/actascitechnol. v38i2.26534

HASSAN, B.G.H.; PING, F. Regional frequency analysis of daily rainfall extremes using L-moments approach. APCBEE Procedia, v.1, p. 126-135, 2012.

KEGGENHOFF, I.; ELIZBARASHVILI, M.; AMINI-FARAHANI, A.; KING, L. Trends in daily temperature and precipitation extremes over Georgia, 1971-2010. Wather and Climate Extremes, v. 4, p. 75-85, 2014.
LIMBERGER, L.; SILVA, M.E.S. Precipitação na bacia amazônica e sua associação à variabilidade da temperatura da superfície dos oceanos Pacífico e Atlântico: uma revisão. Geousp - Espaço e Tempo, v. 20, n. 3, p. 657-675, 2016

MENEZES, F.P.; FERNANDES, L.L.; ROCHA, E.J.P. Uso da estatística para regionalização da precipitação no Estado do Pará, Brasil. Revista Brasileira de Climatologia, v. 16, p. 64-71, 2015.

ONYUTHA, C. Variability of seasonal and anual rainfall in the River Nile riparian countries and possible linkages to ocean-atmosphere interactions. Hydrology Research, v. 47, p. 171-184, 2016.

ORGANIZACIÓN METEOROLÓGICA MUNDIAL (OMM). Guia de Prácticas Hidrológicas. Aquisicion y Proceso de Dados, n. 168, 4a edição, Genebra, 1984. https://hydrologie.org/BIB/OMM/WMOSPA_v5.pdf

ROUSSEEUW, P.J. Silhouettes: a graphical aid to the interpretation and validation of cluster analysis. Journal of Computational and Applied Mathematics, v. 20, p. 53-65, 1987.

SALVIANO, M.F.; GROPPO, J.D.; PELLEGINO, G.Q. Análise de Tendências em Dados de Precipitação e Temperatura no Brasil. Revista Brasileira de Meteorologia, v.31, n. 1, p. 64-73, 2016.

SANTOS, C.; ARAUJO, I.; WANZELER, R.; SERRÃO. E.; FARIAS, M.; LIMA, A. Regionalização hidroclimatológica da bacia hidrográfica do Rio Tapajós. Revista Geografia Acadêmic, v. 9, p. 32-51, 2015 (a).

SANTOS, E.B.; LUCIO, P.S.; SILVA, C.M.S. Precipitation regionalization of the Brazilian Amazon. Atmospheric Science Letters, v.16, p. 185-192, 2015 (b).

SEN, P.K. Estimates of the Regression Coefficient Based on Kendall's Tau. Journal of the American Statistical Association, v. 63, n. 324. p. 1379-1389, 1968.

SHIRIN, AH S.; THOMAS, R. Regionalization of Rainfall in Kerala State. Procedia Tecnologia, v. 24, p. 15-22, 2016.

SILVEIRA, R.N.P.O.; FERNANDES, L.L. e SILVA, M.N.A. Tendência de precipitações pluviométricas e avaliação da influência dos ENOS no distrito de Abunã, Rondônia. Revista Brasileira de Gestão Ambiental, v. 11, n. 1, p. 14-20, 2017.

SOUSA-JÚNIOR, W.C. Tapajós: Hidrelétricas, infraestrutura e caos. Elementos para a governança da sustentabilidade em uma região singular, 1.ed. São José dos Campos: ITA/CTA, p. 92, 2014.

WEBBER, S.R.; WILMOTT, C.J. South American precipitation: 1960-1990 gridded monthly time series (version 1.02), 1998. http://climate.geog.udel.edu/climate.

This is an Open Access article distributed under the terms of the Creative Commons Attribution Non-Commercial License which permits unrestricted non-commercial use, distribution, and reproduction in any medium provided the original work is properly cited. 